\title{
ATOPIC STATUS PROTECTS FROM SEVERE COMPLICATIONS OF COVID-19
}

\author{
Enrico Scala ${ }^{1}$, Damiano Abeni ${ }^{1}$, Alberto Tedeschi ${ }^{2}$, Giuseppina Manzotti $^{3}$, Baoran Yang $^{4}$, \\ Paolo Borrelli ${ }^{5}$, Mauro Giani ${ }^{6}$, Alessandro Marra ${ }^{7}$, Antonio Sgadari ${ }^{8}$, Francesca \\ Saltalamacchia $^{2}$, and Riccardo Asero ${ }^{9}$ \\ ${ }^{1}$ IDI - IRCCS \\ ${ }^{2}$ Ospedale Bolognini di Seriate \\ ${ }^{3}$ Casa di Cura Palazzolo \\ ${ }^{4}$ Azienda Ospedaliera Carlo Poma \\ ${ }^{5}$ Ospedale Beauregard \\ ${ }^{6}$ Istituto Dermopatico dell'Immacolata IRCCS \\ ${ }^{7}$ ASST-Rhodense, PO Rho \\ ${ }^{8}$ Istituto Dermopatico dell'Immacolata Istituto di Ricovero e Cura a Carattere Scientifico \\ ${ }^{9}$ San Carlo Clinic
}

June 1, 2020

\begin{abstract}
BACKGROUND: Severe Covid-19 is associated with a cytokine storm leading to the hyper-expression of cytokines such as IFN-gamma, TNF-alpha, and IL-6, which may be responsible for most severe symptoms and signs of the disease. The immune response of the atopic patient is mostly Th2-oriented, associated with the expression of cytokines such as IL-4, IL-5, and IL-13, and atopic patients express fewer ACE-2 receptors than non-atopic individuals do. We assessed whether atopic status may protect from the most severe consequences of Covid-19. METHODS: Atopic status along with co-factors such as diabetes, hypertension, coronary heart disease, and thrombosis was investigated in severe Covid-19 patients admitted to different Italian hospitals. Patients were classified as having a mild, severe, or very severe disease based on the need of respiratory assistance; severity was plotted against the different co-factors and underwent multivariate analysis. RESULTS: 531 adults aged 25 - 100 years were studied; $57(11.7 \%)$ were atopic. Atopic status showed a significant association with a milder disease irrespective of all other co-factors considered ( $\mathrm{p}<0.001$ ); the protective effect of atopy was detectable throughout all age groups ( $<<0.001)$. CONCLUSIONS: Atopic status appears to protect from the most severe consequences of Covid-19.
\end{abstract}

\section{INTRODUCTION}

One of the most impressive aspects of SARS-CoV-2 infection is the broad spectrum of consequences that may vary from a complete absence of symptoms (asymptomatic infection) to a mild upper airways disease to pneumonia whose severity may range from benign to fatal (1). The most severe complication of the infection by SARS-CoV-2 is the acute respiratory distress syndrome (ARDS) which may lead to mechanical ventilation and ICU admission and is often fatal. The most frequent sign of evolution towards respiratory failure or ARDS is dyspnea associated with decreased oxygenation. The median time from the onset of symptoms to dyspnea is 8 days (2), and the unfavorable evolution occurs about 10-15 days after the onset the first signs and symptoms of the infection (3).

Since SARS-CoV-2 is a new virus, the first-line early defense against it is the innate immunity, before 
the adaptive response occurs. Innate immunity is based on both humoral and cellular elements, including Natural Killer cells and gamma/delta T cells that kill infected cells, thus limiting viral invasion, and secrete cytokines that cause inflammation and stimulate the adaptive immune response. Most severe cases of Covid19, frequently leading to the death of affected patients, are characterized by the activation of two major biological cascades: the so-called "IL-6 cytokine storm" and a disseminated intravascular cascade in the lung. The mechanisms leading to these events are still incompletely defined, but their coincidence with the rise of the adaptive response suggests that the immune response per se, particularly the adaptive one, may play a role. In effect, Covid-19 patients with agammaglobulinemia recovered without experiencing lung complications (4). Thus, the type of immune response rather than the virus itself seems to lead to the inflammatory events observed in the most severe Covid-19 cases.

Atopic status is the genetic predisposition to produce a Type 2 immune response to environmental antigens that are harmless for non-atopic subjects. Type 2 immunity is characterized by the differentiation of naïve T CD4+ cells towards Th2 effector cells, which is followed by IgE production, eosinophilia, and mast cell activation. Type 2 immune response relies on some keystone cytokines, including interleukin (IL) 4, IL-5, IL-9, and IL-13 $(5,6)$. IL-4 induces the differentiation of naïve Th0 cells to Th2 cells, which in turn induce the isotype switching to IgE production. In infection, the Th2 immune response counteracts the microbicidal Th1 response, which could limit the tissue damage induced by Th1-mediated inflammation (7). In a study on experimental Coronavirus 229E infection of the upper airways carried out in healthy volunteers, atopy appeared to be associated with a more severe rhinitis score, suggesting a less efficient anti-virus response (8). Another recent, important finding is the reduced expression of ACE2, the SARS-CoV-2 receptor, in atopic subjects, which could be associated with reduced susceptibility to the virus (9).

Since it seems that the immune response itself, rather than the virus, leads to those catastrophic inflammatory events occurring in the most severe Covid-19 cases, we hypothesized that atopic subjects infected by SARSCoV-2 might have a milder clinical course than non-atopic subjects, and tested this hypothesis in a large cohort of hospitalized Covid-19 patients.

\section{METHODS \\ PATIENTS}

We performed a retrospective study on patients who presented at the ER service of several Italian hospitals with symptoms suggestive of atypical pneumonia and were subsequently hospitalized with a diagnosis of SARS-CoV-2 infection based on the detection of viral nucleic acid in nasal and/or pharyngeal clinical specimens. Most patients were admitted in Covid-19 centers sited in Lombardy (the Italian region most heavily affected by Covid-19), while the remaining patients came from centers in other Italian regions, including Lazio and Aosta Valley. The doctors working in these centers recorded the clinical data of their inpatients with specific reference to respiratory allergy and graded the severity of their respiratory disease at the end of their hospital stay. Patients were considered "atopic" if they had an unequivocal history of respiratory allergy to seasonal and/or perennial airborne allergens confirmed by skin prick tests carried out in a specialist allergy center and/or by elevated specific IgE. Age, sex, and smoking habits were recorded along with several co-morbidities including diabetes, hypertension, coronary heart disease, and thrombosis. The respiratory consequences of the viral infection were classified as mild, severe, or very severe based on no need for respiratory assistance, need for non-invasive respiratory assistance or need for invasive respiratory assistance or death, respectively. Patients' data were anonymized, and the internal review board of the promoting center approved the study.

\section{STATISTICS}

The association between severity of COVID-19 and both the atopy status and the clinical co-factors recorded was studied in both univariate and multivariate analyses. Each variable of interest was dichotomized as negative or positive to study the proportion of subjects with a given clinical status. Categorical variables were analyzed using the Pearsons' $\chi^{2}$ or Fisher's exact test when indicated. Multiple logistic regression was performed to estimate the degree of association of the main exposure variable (i.e., atopy) with COVID-19 
severity after simultaneously adjusting for all the other variables of interest. $\mathrm{P}$ values $<0.05$ were considered significant.

Relatedness of COVID-19 disease grading was tested by applying unsupervised Eisen's hierarchical cluster methods to the data set, encompassing patient' comorbidities and using as agglomeration rule the average linkage clustering as implemented in the program Genesis 1.7.2 (10,11). Unsupervised clustering involved the sorting of both COVID-19 clinical status and patients comorbidities. The COVID-19 disease grading tree was computed based on a full data set and the distance between samples was computed by using the Pearson correlation as similarity measures. As a result, coexistent clinical comorbidities were grouped as hierarchical clusters and presented as heat-maps. Each square in the heat-map represents the presence (red) or the absence (black) of any given tested comorbidity.

The SPSS/PC+ statistical package for statistical evaluation (SPSS, version 15, Chicago, IL) was used to analyze the data.

\section{RESULTS}

\section{Study Population}

531 adult individuals (214 females, 40\%) mean age $68 \pm 14$ years (range 25 - 100 years) with fever, cough, and shortness of breath confirmed as having SARS-CoV-2 infection were studied. Their disease was classified as mild in 191 cases (36\%) and severe or very severe in 340 patients (64\%). The demographic and clinical features of the study population are summarized in table 1.

Male gender was significantly associated with severe disease ( $71 \%$ of males vs $53.7 \%$ of females, $\mathrm{p}=0.001$ ), and consequently, men were more likely to be admitted to intensive care units $(18.3 \%$ vs $10.3 \%$ in female, $\mathrm{p}$ $=0.01$ ).

Smokers had a significantly higher prevalence of coronary heart disease $(35.2 \%$ vs $24.1 \%, \mathrm{p}=0.023)$, and intensive care admission $(22.9 \%$ vs $14.1 \%, \mathrm{p}=0.032)$ than non-smokers. Male smokers showed an even higher prevalence of coronary heart disease $(39.7 \%$ vs $21.6 \%$ in female, $\mathrm{p}=0.002)$ and thrombosis $(19.2 \%$ vs 9.5 , $\mathrm{p}=0.027$ ) than female smokers. (table 1).

\section{Atopic status}

Fifty-seven participants (10.7\% of the study population) were atopic and showed a history of allergy to seasonal and/or perennial airborne allergens (either rhino-conjunctivitis or bronchial asthma). Of these, 32 $(56.1 \%)$ were females (mean age $62 \pm 14$, range 28-93 years). Atopic subjects were younger than non-atopic patients were $(69 \pm 14$ years, $\mathrm{p}=0.01)$, and showed a lower prevalence of coronary heart disease $(\mathrm{p}<0,050)$. Notably, atopic subjects showed a much lower occurrence of severe or very severe COVID-19-related pulmonary involvement ( $33.3 \%$ vs $67.7 \%$ in non-allergic individuals, $\mathrm{p}<0.0001$ ), and therefore a milder disease course. The protective effect of atopic status against severe lung disease was evident throughout all the age subset evaluated, particularly in patients below 60 years of age $(\mathrm{p}=0.001)$ (Figure 1$)$. Intriguingly, a reduced cumulative incidence of death was observed among atopic subjects $(8.8 \%$ vs $18.9 \%$; $=0.059)$, albeit it did not reach statistical significance due to the small number of observations. The multiple logistic regression analysis was first performed including as potential confounders age, sex, smoking, and all the comorbidities. Since none of the comorbidities did contribute to the estimates, we excluded them from the final model, while we decided to still keep age. The adjusted estimate confirmed a significant association between atopic status and reduced severity of COVID-19: in fact, while adjusting for sex, age, and smoking status, non-atopic patients had a significantly higher risk of having severe Covid-19 $\left(\mathrm{OR}_{\text {adj }} 3.0,95 \%\right.$ confidence interval 1.6-5.7, $\mathrm{p}=0.001)($ Table 1$)$.

An unsupervised two-way hierarchical clustering analysis yielded several original patterns of associated clinical features in our population. The simultaneous evaluation of distinct comorbidities at the single patient level generated by the multiple logistic regression analysis identified a clear cluster between mild COVID19 -related pneumonia and atopy status, which were strictly associated $(\mathrm{p}<0.0001 ; \mathrm{ORa}=4.523,95 \% \mathrm{CI}=$ 


\subsection{1-9.221) (Figure 2).}

\section{DISCUSSION}

Several clinical observations demonstrate that the severity of SARS-CoV2 infection may range from asymptomatic to a respiratory illness of variable severity. One of the most serious complications is atypical pneumonia. Critically ill, immunocompetent patients show an uncontrolled secretion of pro-inflammatory cytokines (the so-called cytokine storm) that plays a major role in determining lung injury and are responsible for the high mortality rate. Thus, in severe cases, the virus induces a marked dysregulation of the immune response, which, notably, occurs in immunocompetent individuals. Hyper-expressed cytokines include IFN-gamma, TNF-alpha, and IL-6, which are responsible for symptoms and signs such as fever, fatigue, flu-like symptoms, vascular leakage due to endothelial dysfunction, cardiomyopathy, hypotension, lung injury, activation of the coagulation cascade, and diffuse intravascular coagulation (12-17). The cytokine storm leads to the rapid proliferation and activation of $\mathrm{T}$ cells, macrophages, and natural killer cells that eventually secrete $>150$ inflammatory cytokines, chemokines, and chemical mediators $(18,19)$. The cytokine storm does not occur in patients with uncomplicated SARS-CoV-2 infection $(2,20)$.

Atopic patients are genetically predisposed to mount Th2 type immune-mediated responses; these responses do not imply the expression of the main cytokines involved in the SARS but rather of IL-4, IL-5, and IL-13. Further, a recent study observed a lower expression of the ACE-2 receptor among allergic subjects (9). On this basis, we hypothesized that allergic patients might be both less prone to SARS-CoV-2 infection and/or might have a less severe SARS-CoV-2 infection than non-atopic individuals. To test these two hypotheses, a large epidemiological study on a representative sample including both infected and non-infected subjects considering atopic status as a variable would be required. In the impossibility to carry out such a field epidemiological study, we tried to address the second hypothesis focusing on more than 500 patients with confirmed Covid-19 infection severe enough to warrant hospital admission. Thus, our study population lacks both asymptomatic and symptomatic SARS-CoV-2 infected patients with very mild disease, as these patients had to spend their quarantine period at home. Despite these obvious limitations, our study demonstrates that among hospitalized patients with severe Covid-19, atopic patients have less severe disease. Notably, the "protective" effect of atopic status did not depend on the age or sex of patients nor the presence of other co-factors, such as cigarette smoke, coronary heart disease, diabetes, thrombosis, or hypertension. We are not in the position to ascertain whether the immunological scenario that we have hypothesized and that prompted us to perform this study is correct, as this would require an immune-histochemical analysis of patients' sputum. Nonetheless, our clinical findings make our initial hypothesis believable suggesting that the genetic predisposition to a Th2-oriented immune response might help to avoid the cytokine storm.

In conclusion, atopic status seems to protect against the most severe, often fatal consequences of SARS-CoV2 infection. Such finding may be of help for future studies investigating how to limit the clinical consequences of this infection.

CONFLICT OF INTEREST: No author has conflicts of interest to declare.

FUNDING: no funding.

AUTHORS CONTRIBUTIONS: RA and ES conceived the study. RA coordinated the study and wrote the manuscript. AT, GM, BY, PB, AM, MG, AS, and FS cared for Covid-19 patients and provided the clinical data. DA performed the statistical analysis.

\section{REFERENCES}

1. Zhu N, Zhang D, Wang W, Li X, Yang B, Song J, et al. A Novel Coronavirus from Patients with Pneumonia in China, 2019. N Engl J Med. 2020. [e-pub] DOI:10.1056/NEJMoa2001017.

2. Huang C, Wang Y, Li X, Ren L, Zhao J, Hu Y. Clinical features of patients infected with 2019 novel coronavirus in Wuhan, China. Lanc et 2020; 395: 497-506. doi.org/10.1016/S01406736(20)30183-5.

3. Zhou F, Yu T, Du R, Fan G, Liu Y, Liu Z, et al. Clinical course and risk factors for mortality of adult inpatients with COVID-19 in Wuhan, China: a retrospective cohort study. Lancet 2020; 395:1054-62. 
4. Soresina A, Moratto D, Chiarini M, Paolillo C, Baresi G, Focà E, et al. Favorable outcome of COVID19 in two patients with X-linked agammaglobulinemia. Pediatric Allergy Immunol 2020; doi.org/10.1111/pai.13263

5. Oliphant CJ, Barlow JL, McKenzie AN. Insights into the initiation of type 2 immune responses. Immunology. 2011; 134: 378-385.

6. Wynn TA. Type 2 cytokines: mechanisms and therapeutic strategies. Nat Rev Immunol. 2015; 15 : 271-282.

7. Berger A. Th1 and Th2 responses: what are they? BMJ. 2000;321(7258):424

8. Callow KA, Tyrrell DA, Shaw RJ, Fitzharris P, Wardlaw AJ, Kay AB. Influence of Atopy on the Clinical Manifestations of Coronavirus Infection in Adult Volunteers. Clin Allergy. 1988; 18: 119-29.

9. Jackson JJ, Busse WW, Bacarier LB, Kattan M, O'Connor GT, Wood RA, et al. Association of respiratory allergy, asthma and expression of the SARS-CoV-2 receptor, ACE2. J Allergy Clin Immunol 2020, in press.

10. Eisen MB, Spellman PT, Brown PO, Botstein D: Cluster analysis and display of genome-wide expression patterns. Proc Natl Acad Sci U S A 1998, 95:14863-14868

11. Sturn A, Quackenbush J, Trajanoski Z. Genesis: cluster analysis of microarray data. Bioinformatics 2002; 18:207-8.

12. Shimabukuro-Vornhagen A, Godel P, Subklewe M, et al. Cytokine release syndrome. J Immunother Cancer 2018; 6: 56 .

13. Tanaka T, Narazaki M, Kishimoto T. Immunotherapeutic implications of IL-6 blockade for cytokine storm. Immunotherapy 2016; 8: 959-70.

14. Hunter CA, Jones SA. IL-6 as a keystone cytokine in health and disease. Nat Immunol 2015; 16: 448-57

15. Pathan N, Hemingway CA, Alizadeh AA, et al. Role of interleukin 6 in myocardial dysfunction of meningococcal septic shock. Lancet 2004; 363: 203-9.

16. Hay KA, Hanafi LA, Li D, et al. Kinetics and biomarkers of severe cytokine release syndrome after CD19 chimeric antigen receptor-modified T-cell therapy. Blood 2017; 130: 2295-306.

17. Wang D, Hu B, Hu C, et al. Clinical Characteristics of 138 Hospitalized Patients With 2019 Novel Coronavirus-Infected Pneumonia in Wuhan, China. JAMA 2020

18. Osterholm MT. Preparing for the next pandemic. N Engl J Med 2005; 352(18): 1839-42.

19. Teijaro JR, Walsh KB, Rice S, Rosen H, Oldstone MB. Mapping the innate signalling cascade essential for cytokine storm during influenza virus infection. Proc Natl Acad Sci U S A 2014; 111(10): 3799-804.

20. Zhe Xu, Lei Shi, Yijin Wang, Jiyuan Zhang, Lei Huang, Chao Zhang, Shuhong Liu, Peng Zhao, Hongxia Liu, Li Zhu, Yanhong Tai, Changqing Bai, Tingting Gao, Jinwen Song, Peng Xia, Jinghui Dong, Jingmin Zhao, Fu-Sheng Wang. Pathological findings of COVID-19 associated with acute respiratory distress syndrome. Lancet Respir Med 2020; 8: 420-22.

Table 1: Demographic and clinical features of the study population. The multiple logistic regression analysis including age, sex, smoking, and all the comorbidities, shows a significant association between non-atopic status and a significantly higher risk of having severe COVID-19-related pneumonia.

\section{Hosted file}

image1.emf available at https://authorea.com/users/328414/articles/455707-atopic-statusprotects-from-severe-complications-of-covid-19

Figure 1: Association between atopic status and mild Covid-19 in different age groups. 


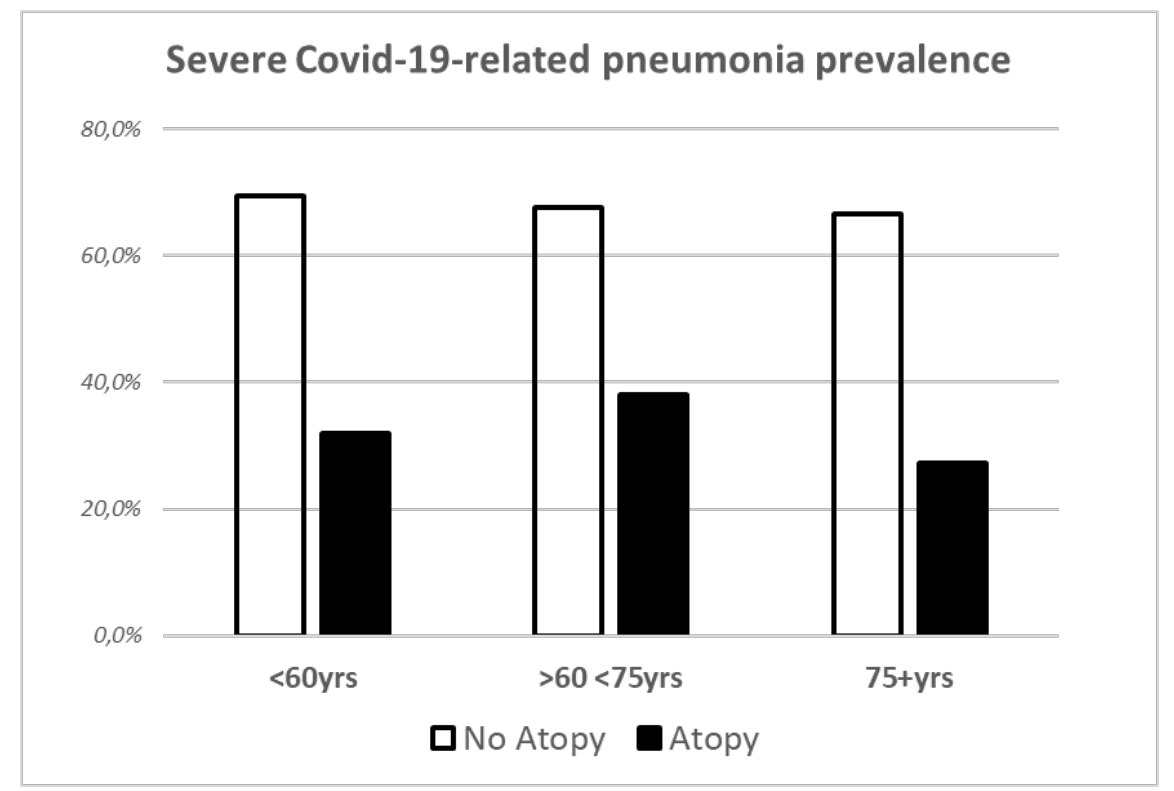

Figure 2. Unsupervised clustering analysis of different co-factors as a function of COVID-19 severity. Atopic status is mainly associated with milder disease outcome.

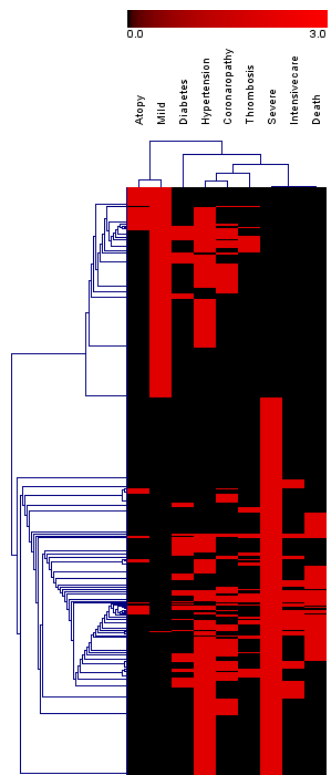

